\title{
The Effectiveness of
} Mindfulness-Based Relapse Prevention on Chinese Methamphetamine Dependent
Patients: A Pilot Study

\author{
Jing Zhai ${ }^{1}$, Yan Long ${ }^{1}$, Jingqing Shi ${ }^{1}$, Daqing Shi ${ }^{1}$, Qihuan Ren ${ }^{1}$, Min Zhao ${ }^{1,2,3 *}$ and \\ Jiang $D u^{1 *}$ \\ ${ }^{1}$ Shanghai Mental Health Center, Shanghai Jiao Tong University School of Medicine, Shanghai, China, ${ }^{2}$ Shanghai Key \\ Laboratory of Psychotic Disorders, Shanghai Jiao Tong University School of Medicine, Shanghai, China, ${ }^{3}$ Chinese Academy \\ of Sciences (CAS) Center for Excellence in Brain Science and Intelligence Technology (CEBSIT), Chinese Academy of \\ Sciences, Shanghai, China
}

OPEN ACCESS

Edited by:

Xiaochu Zhang,

University of Science and Technology

of China, China

Reviewed by:

Chencheng Zhang,

Shanghai Jiao Tong University, China

Mei Yang,

Shenzhen Mental Health

Centre, China

Liu Tieqiao,

Central South University, China

*Correspondence: Jiang Du

dujiangdou@163.com

Min Zhao

drminzhao@smhc.org.cn

Specialty section:

This article was submitted to Addictive Disorders,

a section of the journal

Frontiers in Psychiatry

Received: 20 November 2021

Accepted: 11 January 2022

Published: 28 February 2022

Citation:

Zhai J, Long Y, Shi J, Shi D, Ren Q, Zhao M and Du J (2022) The

Effectiveness of Mindfulness-Based Relapse Prevention on Chinese Methamphetamine Dependent

Patients: A Pilot Study.

Front. Psychiatry 13:819075.

doi: 10.3389/fpsyt.2022.819075
Methamphetamine use is a serious problem in China. Compulsory isolation detoxification is the main treatment measure for drug dependents, whereas psychological interventions in compulsory isolation detoxification centers are extremely inadequate. The current study aimed to examine the effects of mindfulness-based relapse prevention (MBRP) on methamphetamine dependence patients in Chinese compulsory isolation detoxification treatment institutions. Forty-one methamphetamine dependent patients received 16-sessions of MBRP in 8 weeks and assessments were conducted the baseline, 4-, 8-week (after the whole intervention). Results of repeated measured ANOVAs showed there was no significant effect on emotions and cravings. Findings indicated that the effects of MBRP are still difficult to make firm conclusions due to the insignificant results. Future studies should modify the MBRP and ensure that it is suitable for compulsory isolation detoxification treatment institutions in China.

Keywords: mindfulness, intervention, China, methamphetamine, dependence

\section{INTRODUCTION}

Substance use is a serious public health problem and social problem. According to a report by the Chinese government, the number of drug users in China has reached 1.801 million in 2020,57.2\% of them abuse synthetic drugs such as methamphetamine and ketamine, and methamphetamine abuse remains a serious problem (1). Compared to traditional drugs, methamphetamine showed higher addictive and neurotoxicity which would cause brain dysfunction, psychotic symptoms, cognitive impairment, and high risks of relapse (2). Methamphetamine dependent patients generally showed higher craving, impulsive, and negative emotions, and worse cognitive functions (3-5).

In China, compulsory isolation detoxification is the main treatment measure for drug dependents. Compared with the previous treatment system, compulsory isolation detoxification addresses the importance of psychological intervention among the drug dependents since according to the "Drug Control Law of the People's Republic of China," drug dependents have been defined as both offenders and patients, and psychological interventions should be delivered to help them to keep abstinence. To meet the requirement of "Drug Control Law," we try to find new psychological intervention strategies which are eligible for compulsory isolation treatment sites. 
Mindfulness-based relapse prevention therapy (MBRP) developed by Witkiewitz et al. (6) is one of the psychotherapies targeted at substance use disorders (SUDs). MBRP integrated mindfulness meditation and traditional relapse prevention and aimed to help participants develop a sustainable lifestyle and utilize mindfulness skills as effective coping strategies in the face of high-risk situations to prevent relapse (6).

The effect of MBRP on SUDs has received considerable empirical support (7). Compared to the control group, MBRP could reduce patients' craving for drugs and improve their ability to control their negative emotions which is the high-risk factor for relapse. But whether this intervention is appropriate for Chinese compulsory treatment institutions has not been verified.

The pilot study aimed to examine the effectiveness of MBRP on methamphetamine dependent patients in Chinese compulsory isolation detoxification treatment institutes.

\section{METHODS}

\section{Participants}

Forty-one participants were recruited from the compulsory isolation detoxification treatment center in $<$ city $>$ Shanghai $</$ city $>$, China from April to May in 2019. The inclusion criteria were: (1) met the diagnosis of amphetaminetype substance use disorder in the Diagnostic and Statistical Manual of Mental Disorders 5 (DSM-5); (2) completed physiological detoxification; (3) capable of independent communication; (4) aged 18-60. All participants signed informed consent before entering the study.

\section{Procedure}

This study was designed as a randomized controlled clinical trial and has been registered at ClinicalTrials.gov (ID: NCT03748875). Forty-one participants were randomly divided into the MBRP group $(n=21)$ and the control group $(n=20)$. The MBRP group received 16 sessions of 2 -h MBRP interventions (8 weeks, twice per week), which was developed based on the Mindfulnessbased relapse prevention for addictive behaviors: a clinician guide written by Bowen et al. The intervention was led by a professional mindfulness trainer. The control group received the treatment as usual (TAU). Assessments were conducted at the baseline, after the 8th session (mid-test), and the end of the intervention (posttest). See Figure 1 for the flow chart of the current study and Figure 2 for the detailed content of the intervention.

\section{Assessment Depression}

The Chinese version of the Beck Depression Inventory (BDI) was used to measure depression (8). This scale consists of 21 items scored on a 4-point Likert scale, ranging from 0 to 3 . A higher score represents a higher level of depression. The psychometric properties of BDI were acceptable in Chinese samples (8).

\section{Anxiety}

Participants' anxiety was measured using the Chinese version of the State-Trait Anxiety Inventory (STAI) (9). This scale consists of 40 items scored from 1 to 4 , contains two sub-scales: state anxiety and trait anxiety. A higher score indicates a higher level of anxiety. The Chinese version of STAI has satisfying psychometric properties (9).

\section{Craving}

Craving was measured using the Visual Analog Scale (VAS). The $100 \mathrm{~mm}$ line was used to represent the craving degree from 0 (no craving) to 100 (very strong craving). A higher score indicates a higher level of craving.

\section{Data Analysis}

Data were processed with SPSS 21.0. $\chi^{2}$ test and independent $t$-test were used to examine group differences at the baseline. Emotions and craving measured at the baseline, mid-test, and post-test were submitted to 3 (Time) $\times 2$ (Group) repeated measures ANOVAs to examine the effects of the intervention. The significant level was set at 0.05 .

\section{RESULTS}

\section{Baseline Characteristics}

The results of the $\chi^{2}$-test and independent $t$-tests indicated no significant difference between the two groups at the baseline except depression ( $p s>0.05$ ). The BDI scores in the MBRP group were significantly higher than that in the control group at the baseline $\left[t_{(39)}=2.88, p=0.006\right]$. Thus, depression was regarded as a covariate in the following analyses. See Table 1 for more details.

\section{Intervention Effects}

Five participants dropped out because of private reasons. The remaining data from 36 participants were included in further analyses. The results of repeated measured ANOVAs showed that the main effects of group on BDI and state anxiety were significant $\left[F_{(1,34)}=4.890, p=0.034, \eta_{\mathrm{p}}^{2}=0.126\right.$, power $=0.575$; $F_{(1,34)}=4.445, p=.043, \eta_{\mathrm{p}}^{2}=0.119$, power $\left.=0.535\right]$. The further pairwise comparison showed that the BDI scores in the MBRP group were significantly higher than that in the control group $(p=0.034)$, the score of state anxiety in the MBRP group was significantly lower than that in the control group $(p=0.043)$. Moreover, time showed a significant main effect on BDI $\left[F_{(2,34)}\right.$ $=5.271, p=0.007, \eta_{p}^{2}=0.134$, power $\left.=0.819\right]$. The BDI scores at the post-test were significantly lower than that at the baseline $(p=0.009)$ and the mid-test $(p=0.020)$. However, no significant interaction effects of time and groups were found ( $p s>0.05)$. See more details in Table 2 .

\section{DISCUSSION}

This pilot study initially examined the effectiveness of MBRP on methamphetamine dependent patients in Chinese compulsory isolation detoxification treatment institutes. We focused on the effects of MBRP on patients' emotions and cravings in the present study.

The main effect of time on BDI is significant, which indicated that the depression level of methamphetamine dependent patients declined over time. Previous studies found that in the 


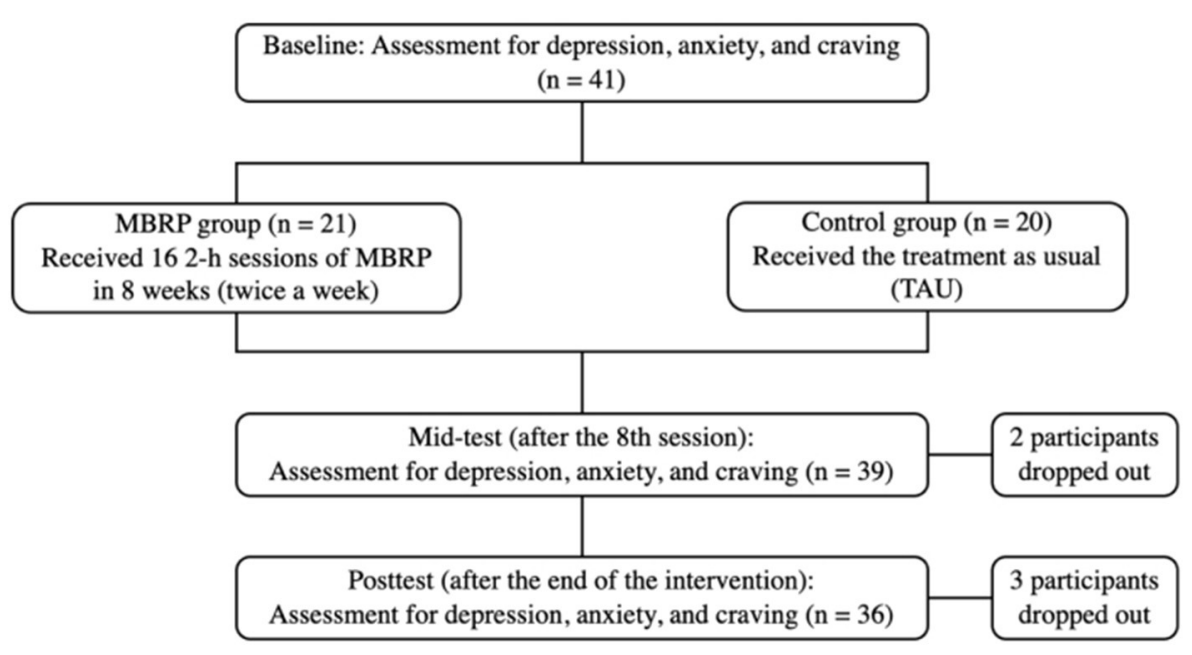

FIGURE 1 | The flow chart of the current study.

\begin{tabular}{|l|l|l|}
\hline Session & \multicolumn{1}{|c|}{ Theme } & \multicolumn{1}{c|}{ Main Practice } \\
\hline Week1 & Automatic navigation and relapse & Body scan, Mindfulness eating \\
\hline Week2 & Triggers and our awareness of the craving & Surfing with the craving, Mountain meditation \\
\hline Week3 & Mindfulness in our daily life & Sitting meditation (hear, breathe), SOBER \\
\hline Week4 & Mindfulness in the high-risk situations & Sitting meditation (see, walk), SOBER \\
\hline Week5 & Acceptance and sensible response & Sitting meditation, Mindfulness stretch, SOBER \\
\hline Week6 & Thought is just a thought & Sitting meditation (thought), SOBER \\
\hline Week7 & Self-care and develop your lifestyle & Loving kindness meditation \\
\hline Week8 & Social support and continued practice & Body scan, SOBER, Final meditation \\
\hline
\end{tabular}

FIGURE 2 | The main content of each intervention sessions.

rehabilitation period, the depression level of methamphetamine dependent patients improved during the time course, which was consistent with the current findings (10). Most notably, the interactions between time and group were not significant on emotions and craving. These results indicated that MBRP showed no significant improved effects on patients' depression, anxiety, and craving. Previous studies generally suggested that MBRP is effective for SUDs to improve emotions and reduce cravings (11). Glasner et al. (12) proved that MBRP could significantly reduce stimulant abuse patients' depression. Similarly, Zullig et al. (13) found that MBRP showed considerable effects on improving opioid use patients' anxiety.

However, numerous studies have suggested that the MBRP intervention has no significant effects on the improvement of emotions and cravings among SUDs. Previous research observed no significant effects of MBRP on anxiety and craving
(13). According to Zullig et al. (13), current results may be at least partially explained by the goals of MBRP are to increase the awareness of cravings and reduce behavioral and emotional reactivity to cravings, but not to eliminate the cravings themselves.

Regarding the current study, it was conducted in the compulsory isolation detoxification treatment institutes, in which patients' daily schedules were strictly arranged. Thus, the lacking meditation practice might reduce the intervention effect. Another impacting factor is the changing motivation. The participants in the compulsory isolation detoxification treatment institutes are relatively lacking in changing motivation, which might be an important reason for the insignificant effect of MBRP intervention in the current study (14). Other factors include small sample size, gender bias (all males), which will affect the research findings to a certain extend. More importantly, previous studies 
TABLE 1 | Baseline comparison of demographic variables, substance use situations, and all outcome variables between the MBRP group and the control group before the intervention $[M \pm S D / n(\%)](N=41)$.

\begin{tabular}{|c|c|c|c|c|}
\hline & $\begin{array}{l}\text { MBRP group } \\
\quad(n=21)\end{array}$ & Control group $(n=20)$ & $\chi^{2} / \mathrm{t}$ & $p$ \\
\hline Age & $35.38 \pm 6.045$ & $38.80 \pm 9.395$ & -1.393 & 0.172 \\
\hline Marriage & 0.299 & 0.585 & & \\
\hline Married & 8 (38.1\%) & $6(30.0 \%)$ & & \\
\hline Single or divorced & $13(61.9 \%)$ & $14(70.0 \%)$ & & \\
\hline Years of education & $11.05 \pm 3.43$ & $12.40 \pm 2.96$ & -1.349 & 0.185 \\
\hline Occupation & 0.811 & 0.368 & & \\
\hline Employed & $13(61.9 \%)$ & $15(75.0 \%)$ & & \\
\hline Unemployed & 8 (38.1\%) & 5 (25.0\%) & & \\
\hline Months used accumulatively & $29.88 \pm 49.67$ & $37.21 \pm 54.94$ & -0.443 & 0.660 \\
\hline Compulsory isolation times & 0.786 & 0.675 & & \\
\hline Once & $16(76.2 \%)$ & $13(65 \%)$ & & \\
\hline Twice or three times & $3(14.3 \%)$ & $5(25 \%)$ & & \\
\hline More than three times & $2(9.5 \%)$ & $2(10 \%)$ & & \\
\hline $\mathrm{BDI}$ & $19.29 \pm 7.23$ & $13.10 \pm 6.49$ & $2.878^{\star}$ & 0.006 \\
\hline \multicolumn{5}{|l|}{ STAI } \\
\hline State anxiety & $40.62 \pm 9.368$ & $42.15 \pm 8.63$ & -0.543 & 0.590 \\
\hline Trait anxiety & $44.24 \pm 12.03$ & $40.75 \pm 8.78$ & 1.056 & 0.297 \\
\hline VAS craving & $28.67 \pm 27.20$ & $20.3 \pm 26.26$ & 1.001 & 0.323 \\
\hline
\end{tabular}

${ }^{\star} p<0.05$.

TABLE 2 | The results of the repeated measured ANOVAs in all outcome variables $(N=36)$.

\begin{tabular}{|c|c|c|c|c|c|c|c|c|}
\hline & & \multirow{2}{*}{$\begin{array}{l}\text { MBRP group } \\
\quad(n=20)\end{array}$} & \multirow{2}{*}{$\begin{array}{l}\text { Control group } \\
\qquad(n=16)\end{array}$} & \multicolumn{5}{|c|}{ Repeated-Measured ANOVAs } \\
\hline & & & & & $\boldsymbol{F}$ & $p$ & $\eta^{2}$ & Power \\
\hline \multirow[t]{3}{*}{$\mathrm{BDI}$} & Baseline & $19.30 \pm 7.42$ & $12.94 \pm 6.75$ & G & $4.890^{\star}$ & 0.034 & 0.126 & 0.575 \\
\hline & Mid-Test & $16.60 \pm 7.82$ & $13.19 \pm 6.86$ & $\mathrm{~T}$ & $5.271^{\star}$ & 0.007 & 0.134 & 0.819 \\
\hline & Post-test & $14.90 \pm 9.09$ & $10.12 \pm 7.00$ & $\mathrm{G} \times \mathrm{T}$ & 0.854 & 0.43 & 0.025 & 0.191 \\
\hline \multicolumn{9}{|l|}{ STAI } \\
\hline \multirow[t]{3}{*}{ State anxiety } & Baseline & $40.85 \pm 9.55$ & $43.125 \pm 9.14$ & G & $4.445^{\star}$ & 0.043 & 0.119 & 0.535 \\
\hline & Mid-Test & $41.10 \pm 9.23$ & $43.875 \pm 12.17$ & $\mathrm{~T}$ & 1.592 & 0.211 & 0.046 & 0.326 \\
\hline & Post-test & $40.30 \pm 10.62$ & $43.75 \pm 12.87$ & $G \times T$ & 0.710 & 0.495 & 0.021 & 0.165 \\
\hline \multirow[t]{3}{*}{ Trait anxiety } & Baseline & $44.40 \pm 12.31$ & $41.4375 \pm 9.37$ & $G$ & 2.365 & 0.134 & 0.067 & 0.321 \\
\hline & Mid-Test & $42.15 \pm 9.89$ & $42.44 \pm 10.70$ & $\mathrm{~T}$ & 1.050 & 0.356 & 0.031 & 0.226 \\
\hline & Post-test & $41.8 \pm 10.78$ & $42.19 \pm 9.11$ & $G \times T$ & 1.076 & 0.347 & 0.032 & 0.231 \\
\hline \multirow[t]{3}{*}{ VAS } & Baseline & $29.85 \pm 27.34$ & $19.063 \pm 25.91$ & $G$ & 0.358 & 0.554 & 0.011 & 0.089 \\
\hline & Mid-Test & $30.05 \pm 30.72$ & $20.19 \pm 30.549$ & $\mathrm{~T}$ & 1.320 & 0.274 & 0.038 & 0.276 \\
\hline & Post-test & $21.65 \pm 25.29$ & $16.56 \pm 23.301$ & $G \times T$ & 0.714 & 0.494 & 0.021 & 0.166 \\
\hline
\end{tabular}

G, group; T, time; * $p<0.05$.

The reviewer CZ declared a shared affiliation, with no collaboration, with the authors JZ, YL, JS, DS, QR, MZ, and JD at the time of the review.

found that methamphetamine showed higher neurotoxicity and addiction (2), which might also affect the effectiveness of the MBRP intervention. Further studies could conduct in a larger sample size and combine other techniques (such as virtual realit or motivation intervention) with MBRP to improve the effectiveness of MBRP in methamphetamine dependent patients.

Even the results were negative, the present study still provides preliminary evidence for the implementation of MBRP on methamphetamine patients. Given the specific settings for compulsory rehabilitation treatment in China, it might be necessary to modify MBRP and ensure it is suitable for the current situation.

Limitations to this study include assessing all data via selfreport scales which might be impacted by social desirability bias, all the included methamphetamine dependent patients are male while gender might influence the vulnerability to methamphetamine toxicity, and lack of biological indicators as the biological improvements might not be reflected in behaviors 
$(15,16)$. It would be worthwhile to conduct further study in a larger sample using comprehensive measurements, such as the behavioral tasks to expand the result.

\section{DATA AVAILABILITY STATEMENT}

The raw data supporting the conclusions of this article will be made available by the authors, without undue reservation.

\section{ETHICS STATEMENT}

The studies involving human participants were reviewed and approved by the Institutional Review Boards of the Shanghai Mental Health Center. The patients/participants provided their written informed consent to participate in this study.

\section{AUTHOR CONTRIBUTIONS}

JD and MZ contributed to the conceptualization and design of the study. JS, DS, and QR collected the data. YL performed the statistical analysis and the interpretation of data. JZ and YL

\section{REFERENCES}

1. CNNCC. 2019 Report on Drug Situation in China. CNNCC-China National Narcotics Control Commission (2020). Available online at: http://www. nncc626.com/2020-06/24/c_1210675813.htm

2. Courtney KE, Ray LA. Methamphetamine: an update on epidemiology, pharmacology, clinical phenomenology, treatment literature. Drug Alcohol Depend. (2014) 143:11-21. doi: 10.1016/j.drugalcdep.201 4.08 .003

3. Darke S, Kaye S, McKetin R, Duflou J. Major physical and psychological harms of methamphetamine use. Drug Alcohol Rev. (2008) 27:25362. doi: 10.1080/09595230801923702

4. Pan K, Li R. Psychological craving and its influence factors in male heroin and methamphetamine addicts. J Psychiatry. (2018) 31:301-3. doi: 10.3969/j.issn.2095-9346.2018.04.017

5. Ding $\mathrm{X}$, Zhao M. Research progress on cognitive impairments in methamphetamine patients. J Psychiatry. (2020) 33:66-9. doi: 10.3969/j.issn.2095-9346.2020.01.017

6. Witkiewitz K, Marlatt G, Walker D. Mindfulness-Based relapse prevention for alcohol and substance use disorders. J Cogn Psychother. (2005) 19:21128. doi: 10.1891/jcop.2005.19.3.211

7. Grant S, Colaiaco B, Motala A, Shanman R, Booth M, Sorbero M, et al. Mindfulness-based relapse prevention for substance use disorders: a systematic review and meta-analysis. Journal of Addict Med. (2017) 11:38696. doi: 10.1097/ADM.0000000000000338

8. Wang Z, Yuan C, Huang J, Li Z, Chen J, Zhang H, et al. Reliability and validity of the Chinese version of beck depression inventoryI among depression patients. Chin Ment Health J. (2011) 25:476-9. doi: 10.3969/j.issn.1000-6729.2011.06.014

9. Li W, Qian M. Revision of the norm of state-trait anxiety inventory (STAI) for Chinese college students. Acta Sci Nat Univer Pekinensis. (1995) 31:109.

10. Polcin DL, Witbrodt J, Korcha R, Gupta S, Mericle AA. Course of psychiatric symptoms and abstinence among methamphetamine-dependent persons in sober living recovery homes. J Psychoact Drugs. (2016) 48:17380. doi: 10.1080/02791072.2016.1180466

11. Bowen S, Chawla N, Collins SE, Witkiewitz K, Hsu S, Grow $\mathrm{J}$, et al. Mindfulness-Based relapse prevention for substance use disorders: a pilot efficacy trial. Subst Abuse. (2009) 30:295305. doi: $10.1080 / 08897070903250084$ drafted the manuscript. JD provided scientific revisions to the manuscript. All authors contributed to the article and approved the submitted version.

\section{FUNDING}

This work was supported by National Nature Science Foundation of China (81871045 and 8217050343), Science and Technology Commission of Shanghai Municipal (19411969200 and 21DZ2201000), Shanghai Municipal Science and Technology Major Project (2018SHZDZX05), Shanghai Clinical Research Center for Mental Health (19MC1911100), Shanghai Engineering Research Center of Intelligent Addiction Treatment and Rehabilitation (19DZ2255200), and Shanghai Key Laboratory of Psychotic Disorders (13DZ2260500).

\section{ACKNOWLEDGMENTS}

The authors thank all the supports from Shanghai Gaojing compulsory isolation detoxification treatment institutes.

12. Glasner S, Mooney LJ, Ang A, Garneau HC, Hartwell E, Brecht L, et al. Mindfulness-Based relapse prevention for stimulant dependent adults: a pilot randomized clinical trial. Mindfulness. (2017) 8:12635. doi: 10.1007/s12671-016-0586-9

13. Zullig KJ, Lander LR, Sloan S, Brumage MR, Hobbs GR, Faulkenberry L. Mindfulness-Based relapse prevention with individuals receiving medicationassisted outpatient treatment for opioid use disorder. Mindfulness. (2018) 9:423-9. doi: 10.1007/s12671-017-0784-0

14. Wu X, Zeng H, Li L, Chen Y. Investigation and analysis of the motivation for abstaining from drugs in 1294 drug addicts. Chin J Drug Depend. (2009) 18:207-14. doi: 10.13936/j.cnki.cjdd1992.2009.03.013

15. Sallis JF, Saelens BE. Assessment of physical activity by self-report: status, limitations, future directions. Res Q Exerc Sport. (2000) 71:S114. doi: 10.1080/02701367.2000.11082780

16. Khajehpour H, Mohagheghian F, Ekhtiari H, Makkiabadi B, Jafari AH, Eqlimi $\mathrm{E}$, et al. Computer-aided classifying and characterizing of methamphetamine use disorder using resting-state EEG. Cogn Neurodyn. (2019) 13:51930. doi: 10.1007/s11571-019-09550-z

Conflict of Interest: The authors declare that the research was conducted in the absence of any commercial or financial relationships that could be construed as a potential conflict of interest.

The reviewer CZ declared a shared affiliation, with no collaboration, with the authors JZ, YL, JS, DS, QR, MZ, and JD at the time of the review.

Publisher's Note: All claims expressed in this article are solely those of the authors and do not necessarily represent those of their affiliated organizations, or those of the publisher, the editors and the reviewers. Any product that may be evaluated in this article, or claim that may be made by its manufacturer, is not guaranteed or endorsed by the publisher.

Copyright (C) 2022 Zhai, Long, Shi, Shi, Ren, Zhao and Du. This is an open-access article distributed under the terms of the Creative Commons Attribution License (CC $B Y)$. The use, distribution or reproduction in other forums is permitted, provided the original author(s) and the copyright owner(s) are credited and that the original publication in this journal is cited, in accordance with accepted academic practice. No use, distribution or reproduction is permitted which does not comply with these terms. 